\title{
Quantum corrections to the phase diagram of heavy-fermion superconductors
}

\author{
André S. Ferreira and Mucio A. Continentino \\ Instituto de Física, Universidade Federal Fluminense, \\ Campus da Praia Vermelha, \\ Niterói, RJ, 24.210-340, Brazil \\ Eduardo C. Marino \\ Instituto de Física, Universidade Federal do Rio de Janeiro, \\ Rio de Janeiro, RJ, 21945-970, Brazil.
}

(Dated: November 2, 2018)

\begin{abstract}
The competition between magnetism and Kondo effect is the main effect determining the phase diagram of heavy fermion systems. It gives rise to a quantum critical point which governs the low temperature properties of these materials. However, experimental results made it clear that a fundamental ingredient is missing in this description, namely superconductivity. In this paper we make a step forward in the direction of incorporating superconductivity and study the mutual effects of this phase and antiferromagnetism in the phase diagram of heavy fermion metals. Our approach is based on a Ginzburg-Landau theory describing superconductivity and antiferromagnetism in a metal with quantum corrections taken into account through an effective potential. The proximity of an antiferromagnetic instability extends the region of superconductivity in the phase diagram and drives this transition into a first order one. On the other hand superconducting quantum fluctuations near a metallic antiferromagnetic quantum critical point gives rise to a first order transition from a low moment to a high moment state in the antiferromagnet. Antiferromagnetism and superconductivity may both collapse at a quantum bicritical point whose properties we calculate.
\end{abstract}

\section{INTRODUCTION}

Competition between superconductivity (SC) and magnetism plays an important role in determining the physical properties of strongly correlated superconducting materials, such as high- $\mathrm{T}_{c}$ superconductors ${ }^{1.2}$ and heavy fermions $3 \cdot 4 \cdot 5 \cdot 6.7$. The study of the phase diagrams of such materials suggests that these phases are intrinsically related. This relation and the interface between these phases are among the fundamental issues that have not yet been clarified in condensed matter physics. In particular, the case of high- $T_{c}$ oxides represents a formidable problem as the nature of the normal phase itself is not understood.

The study of heavy fermion materials has focused until recently on the competition between long range magnetic ordering due to the RKKY interaction and the Kondo effect. However, low temperature experiments in these systems have shown that they can exhibit superconductivity near or in coexistence with an antiferromagnetic $(\mathrm{AF})$ phase close to a quantum critical point $(\mathrm{QCP})^{8}$. This can be conveniently tuned by varying some element concentration or pressure ${ }^{6}$. In distinction with the high$T_{c}$ materials in the case of heavy fermions we have a quite reasonable understanding of their normal phase. Below the coherence line $T_{\text {coh }}$ this is essentially a strongly correlated, nearly antiferromagnetic, Fermi liquid ${ }^{9}$. Above this line and in particular at the $\mathrm{QCP}$, the non-Fermi liquid behavior is well understood in terms of quantum criticality and the associated critical exponents 10 .

In this paper our aim is to investigate the mutual effects of superconductivity and antiferromagnetism at zero temperature and how this determines the phase di- agram of three dimensional heavy fermions $(\mathrm{d}=3)$. Our knowledge of the metallic phase in these materials provides a solid starting point for the approach we use. We consider a Ginzburg-Landau functional which contains the magnetic and superconducting order parameters and their coupling. We apply the effective potential method used in quantum field theory $\frac{11.12}{10}$ calculate the quantum corrections to this classical action. This method is implemented up to one loop order and requires the knowledge of the propagator solutions of the quadratic functionals associated with the decoupled fields. In such approach, the normal, metallic paramagnetic phase is described by the usual dissipative propagator that takes into account the dynamics of the spin fluctuations (paramagnons) in the nearly antiferromagnetic metal ${ }^{13.14}$.

We investigate several phase diagrams in $d=3$. A possible scenario is that of a quantum bicritical point separating a metallic antiferromagnet from a superconducting phase whose universality class has been identified ${ }^{15}$. The model also describes the case where the $\mathrm{AF}$ and $\mathrm{SC}$ transitions occur at distinct quantum critical points (QCPs), so that, there is a normal state between the $\mathrm{AF}$ and $\mathrm{SC}$ phases. The proximity to an AF instability drives the $\mathrm{SC}$ transition to the normal state into a first order one and enlarges the region of the phase diagram where superconductivity exists 15 . On the other hand superconducting fluctuations drive an AF-QCP into a discontinuous transition and give rise to a new AF phase with a reduced value of the order parameter. We present the Ginzburg-Landau model and the calculation of the effective potential in detail since, as far as we know, this method has not been sufficiently explored in condensed matter physics. Although the model refers specifically to 
the coupling between AF and SC order parameters, the results obtained should be considered more general, independent of the specific nature of the instabilities, as long as, the dynamics are similar. For example, a structural instability associated with a soft optical phonon mode has a dynamic description which is similar to that given to the superconductor here.

It is interesting that recent experiments in heavy fermions suggest the existence of intrinsic inhomogeneities in these materials near their magnetic quantum critical point ${ }^{16}$. Our model provides a natural explanation for these inhomogeneities, attributing them to the weak first order transitions and the associated phenomena of phase coexistence and spinodals arising from the quantum corrections due to the coupling between different order parameters.

We point out that similar models describing the competition between superconductivity and antiferromagnetism have been proposed $17,18.19$. The emphasis however has been on the classical aspects of this phenomenon and their aim was to describe high- $T_{c}$ superconductors. The validity of this approach for this class of systems is now subject of intense debate ${ }^{20,21}$.

\section{PARAMAGNON AND SUPERCONDUCTING PROPAGATORS}

The model Ginzburg-Landau action contains three real fields. Two fields, $\phi_{1}$ and $\phi_{2}$, correspond to the two components of the superconductor order parameter (the ground state wave function, for example). The other field $\phi_{3}$, for simplicity represents a one component antiferromagnetic order parameter. The results are immediately generalized to a three component $\mathrm{AF}$ vector field, with the unique consequence of changing some numerical factors as discussed below. In order to include quantum fluctuations by the effective potential method it is convenient to describe the quadratic parts of the action by the associated propagators. The propagator associated with the free, quadratic action of the superconductor can be directly obtained from a quantum GinzburgLandau action ${ }^{22}$. However, finding the appropriate form of the superconductor propagator is a difficult problem since the nature of the fluctuations driving the superconducting transition can change considerably the quadratic part of this generalized action. For metallic host where pair breaking interactions due to magnetic impurities destroy superconductivity the quadratic action has been obtained in Ref ${ }^{23}$ and is associated with a dynamic exponent $z=2$. The case of BCS superconductors in which any attractive interaction $U$ makes the system superconducting at $T=0$ has been studied in Ref ${ }^{24}$. In this case the form of the action directly reflects the fact that the quantum critical point occurs for $U=0$. Here we are interested in pure systems, so that criticality is achieved by pressure and impurities are not considered. Also, in our case, superconductivity is certainly non-BCS and finding the correct propagator is in itself a very difficult problem. In order to make progress, we choose to consider the simplest generalization of the classical Ginzburg-Landau action,

$$
G_{0}(k)=G_{0}(\omega, \boldsymbol{q})=\frac{i}{k^{2}-m^{2}}
$$

where $k$ is a four-vector $(\omega, \boldsymbol{q})$ with $k^{2}=\omega^{2}-q^{2}$ and we took the Fermi wave vector $q_{F}=0$. This propagator assumes the existence of a gap or pseudogap in the excitation spectrum of the phase precursive to the superconducting one. There is plenty of evidence for such pseudogaps in High-Tc superconductors ${ }^{25}$ and also now in heavy-fermions systems as shown in recent experiments ${ }^{26}$. Attractive Hubbard models also show normal phases with charge ordering and a gap for excitations that vanishes in the superconductor quantum critical point 27 .

We work in Minkowsky space since the present superconductor propagator is Lorentz invariant and we want to obtain the effective potential as in quantum field theory, following closely the work of Coleman and Weinberg 12 . It's clear from Eq. (11) that time acts as an extra direction on the same footing as the spatial ones, so that, the associated dynamic exponent is $z=1$.

The quadratic functional associated with the magnetic part represented by the field $\phi_{3}$, the sub-lattice magnetization, takes into account the dissipative nature of the paramagnons near the magnetic phase transition. This approximation to the full paramagnon propagator can be found in detail in the work by Hertz ${ }^{13}$ and here we present a brief derivation. The expansion of the magnetic free-energy functional in a power series of the order parameter can be written as

$$
\begin{aligned}
S_{\text {mag }}\left(\phi_{3}\right)= & \int d^{4} k v_{2}(k)\left|\phi_{3}(k)\right|^{2}+ \\
& +\int d^{4} k_{1} d^{4} k_{2} d^{4} k_{3} d^{4} k_{4} v_{4}\left(k_{1}, k_{2}, k_{3}, k_{4}\right) \phi_{3}\left(k_{1}\right) \phi_{3}\left(k_{2}\right) \phi_{3}\left(k_{3}\right) \phi_{3}\left(k_{4}\right) \delta\left(k_{1}+k_{2}+k_{3}+k_{4}\right)+\ldots
\end{aligned}
$$


susceptibility $\chi$ calculated in RPA is

$$
\operatorname{Im} \chi(\omega, \boldsymbol{q})=\operatorname{Im}\left(\frac{\chi_{0}(\omega, \boldsymbol{q})}{1-U \chi_{0}(\omega, \boldsymbol{q})}\right)
$$

where $\chi_{0}$ is the susceptibility of the free-electron model ${ }^{29}$. This susceptibility gives a good representation of the paramagnon propagator associated with the quadratic coefficient $v_{2}$. If we are near a magnetic instability we can expand $\chi_{0}$ for long wavelengths and low frequencies. Substituting this expansion in the RPA form of the susceptibility Eq. (3) and choosing the units appropriately we can write the AF paramagnon propagator as

$$
D_{0}(\omega, \boldsymbol{q})=\frac{i}{i|\omega| \tau-q^{2}-m_{p}^{2}}
$$

where $\tau$ is a characteristic relaxation time and $m_{p}^{2}$ is related to local Coulomb repulsion $U$ and the density of states at the Fermi level $N\left(E_{F}\right)$ by

$$
m_{p}^{2}=1-U N\left(E_{F}\right) .
$$

The quadratic parts of the effective functional are then completely characterized by the propagators of Eq. (II) and Eq. (4). Notice that the magnetic propagator, Eq. (44), is sufficiently general to be associated with a Hamiltonian which preserves rotational invariance $\frac{13}{3}$. In this case $\phi_{3}$ is replaced by a vector and in the second term of Eq. (2) the quartic interaction is written in the form $\left(\left|\phi_{3}\right|^{2}\left|\phi_{3}\right|^{2}\right)$ as appropriate for vector fields. The use of a vector field modifies the effective potential only by numerical factors as we show in the next sections.

\section{CLASSICAL POTENTIAL}

The classical part of the potential is given by,

$$
\begin{array}{r}
V_{c l}\left(\phi_{1}, \phi_{2}, \phi_{3}\right)=\frac{1}{2} m^{2}\left(\phi_{1}^{2}+\phi_{2}^{2}\right)+\frac{1}{2} m_{p}^{2} \phi_{3}^{2}+ \\
+V_{s}\left(\phi_{1}, \phi_{2}\right)+V_{p}\left(\phi_{3}\right)+V_{i}\left(\phi_{1}, \phi_{2}, \phi_{3}\right)
\end{array}
$$

where the self-interaction of the superconductor field is,

$$
V_{s}\left(\phi_{1}, \phi_{2}\right)=\frac{\lambda}{4 !}\left(\phi_{1}^{2}+\phi_{2}^{2}\right)^{2}
$$

and that of the antiferromagnet is given by,

$$
V_{p}\left(\phi_{3}\right)=\frac{g}{4 !} \phi_{3}^{4}
$$

The coupling $g$ above is related to the coefficient $v_{4}$ in the expansion of Eq. (2) with all dependence on the fourvector $k$ ignored. Finally, the last term is the (minimum) interaction between the relevant fields,

$$
V_{i}\left(\phi_{1}, \phi_{2}, \phi_{3}\right)=u\left(\phi_{1}^{2}+\phi_{2}^{2}\right) \phi_{3}^{2} .
$$

This term is the first allowed by symmetry on a series expansion of the interaction. It is possible that in regions of the phase diagram where phase coexistence between superconductivity and antiferromagnetism occurs higher order terms should be included in the free energy. However, we will not consider the case of coexistence in the present work. Notice that for $u>0$, which is the case here, superconductivity and antiferromagnetism are in competition and the possibility of AF acting as a pair breaking perturbation is considered.

The minima of the classical potential yield the classical ground states of the system 19 . The different possibilities for the phase diagrams described by the classical action at mean field level ${ }^{6,17.19 .30}$ are shown schematically in Fig. 1. The $T=0$ transitions are all continuous, second order phase transitions. Notice that in Eq. (6) there

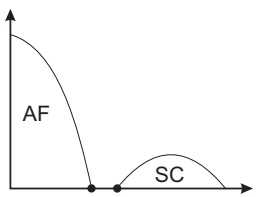

(A)

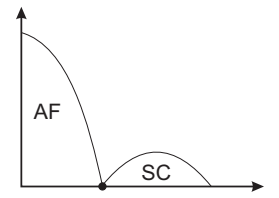

(B)

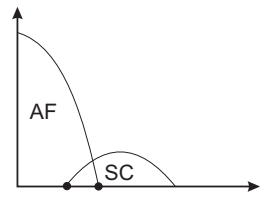

(C)
FIG. 1: Possible classical temperature phase diagrams for a heavy fermion system (schematic). $\mathrm{AF}$ and $\mathrm{SC}$ refer to the superconductor and antiferromagnetic phases respectively.

are two mass terms, $m^{2}$ and $m_{p}^{2}$, which represent respectively, the distance to the superconductor and the antiferromagnetic quantum critical points. These two terms appear in the quadratic parts of the action and are already included in the propagators of Eq. (1) and Eq. (4) to be used in the computation of the effective potential below.

The classical potential can be generalized to a rotational invariant form replacing the scalar field $\phi_{3}$ by a vector. As discussed before the self-interaction is then of the form

$$
V_{p}\left(\phi_{3}\right)=\frac{g}{4 !}\left|\phi_{3}\right|^{2}\left|\phi_{3}\right|^{2}=\frac{g}{4 !} \sum_{i, j} \phi_{3, i}^{2} \phi_{3, j}^{2}
$$

where $i$ and $j$ label the components of the vector. For this generalized potential, however, the minima depend only on the modulus of the vector $\phi_{3}$ and the use of this vector field brings only slight modifications to the effective potential.

In the next sections we obtain quantum corrections to the classical potential. These corrections can modify significantly the physics of the problem and the phase diagrams changing, for example, the order of the transitions.

\section{ONE LOOP EFFECTIVE POTENTIAL}

The first quantum correction to the potential can be obtained by the summation of all one loop diagrams (Fig. 2). 


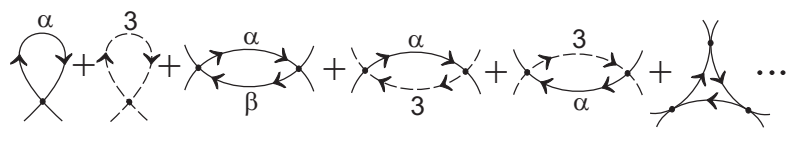

FIG. 2: One loop diagrams. The superconductor fields are represented by $\alpha$ or $\beta=1,2$. The doted line represents the unusual propagator of Eq. (4).

We apply the general method proposed by Coleman ${ }^{31}$ with minimum modifications to account for the different nature of the propagators in our problem. The sum over the field indices can be easily done if we define a vertex matrix $M$, given by

$$
[M]_{l m}=-\left.i K_{0}^{l} \frac{\partial^{2} V_{c l}}{\partial \phi_{l} \partial \phi_{m}}\right|_{\{\phi\}=\left\{\phi_{c}\right\}}
$$

and then take the trace. In Eq. (111) the propagator $\left(K_{0}^{l}=G_{0}\right.$ or $\left.D_{0}\right)$ of Eq. (11) or Eq. (4) is incorporated in the definition of the matrix. We draw the loops with arrows and choose the outgoing propagator of each ver- tex to be included in the associated element. The matrix $M$ is then obtained deriving the classical potential with respect to the fields $\{\phi\}$ and taking the values of these derivatives at the classical values of the fields, $\left\{\phi_{i c}\right\}$. The sum of diagrams with the correct Wick factors is formally done in momentum space and using the property of the trace

$$
\operatorname{Tr}[\ln (1-M)]=\ln \operatorname{det}[(1-M)]
$$

we get

$$
V^{(1)}\left[\phi_{c}\right]=\frac{i}{2} \hbar \int d^{4} k \ln \operatorname{det}[1-M(k)] .
$$

The $3 \times 3$ matrix $M$ can be simplified if we choose the classical minimum of the superconductor fields imposing $\phi_{2 c}=0$ (this can be done because the minimum depends only on the modulus $\phi_{1 c}^{2}+\phi_{2 c}^{2}$ ). Hence, rotating to Euclidean space, so that, $k^{2}=\omega^{2}+q^{2}$ and using $\hbar=1$ units the first quantum correction can be written as

$$
\begin{aligned}
V^{(1)}\left(\phi_{1 c}, \phi_{3 c}\right)= & \frac{1}{2} \int \frac{d^{4} k}{(2 \pi)^{4}}\left\{\ln \left(1+\frac{A\left(\phi_{1 c}, \phi_{3 c}\right)}{k^{2}+m^{2}}\right)+\ln \left[\left(1+\frac{B\left(\phi_{1 c}, \phi_{3 c}\right)}{k^{2}+m^{2}}\right)\left(1+\frac{C\left(\phi_{1 c}, \phi_{3 c}\right)}{|\omega| \tau+q^{2}+m_{p}^{2}}\right)+\right.\right. \\
& \left.\left.-\left(\frac{D^{2}\left(\phi_{1 c}, \phi_{3 c}\right)}{\left(k^{2}+m^{2}\right)\left(|\omega| \tau+q^{2}+m_{p}^{2}\right)}\right)\right]\right\}
\end{aligned}
$$

where

$$
\begin{array}{r}
A\left(\phi_{1 c}, \phi_{3 c}\right)=(\lambda / 6) \phi_{1 c}^{2}+2 u \phi_{3 c}^{2} \\
B\left(\phi_{1 c}, \phi_{3 c}\right)=(\lambda / 2) \phi_{1 c}^{2}+2 u \phi_{3 c}^{2} \\
C\left(\phi_{1 c}, \phi_{3 c}\right)=2 u \phi_{1 c}^{2}+(g / 2) \phi_{3 c}^{2} \\
D\left(\phi_{1 c}, \phi_{3 c}\right)=4 u \phi_{1 c} \phi_{3 c}
\end{array}
$$

The total effective potential with first order quantum corrections is then given by

$$
V_{e f}\left(\phi_{1 c}, \phi_{3 c}\right)=V_{c l}\left(\phi_{1 c}, \phi_{3 c}\right)+V^{(1)}\left(\phi_{1 c}, \phi_{3 c}\right)
$$

where $V_{c l}$ is the classical potential of Eq. (6) and $V^{(1)}$ is the first quantum correction of order $\hbar$ of Eq. (14). In the subsequent sections we consider the effects of these quantum correction for the classical results. We give special attention to the possibility of fluctuation induced symmetry breaking and first order phase transitions at $T=0$. We point out again that the results for an isotropic spin model are similar since, when dealing with a vector magnetic order parameter $\phi_{3}$, we can use the fact that the classical minima depend only on the modulus of this vector. The modifications which arise in the final effective potential are simply different numerical factors coming from new but equivalent terms in Eq. (14).

\section{POSSIBLE PHASE DIAGRAMS}

The ground state phase diagram of a heavy-fermion with $\mathrm{AF}$ and SC phases can be obtained from the classical action by varying the masses $m$ and $m_{p}$. The normal paramagnetic state has $m_{p}^{2}>0$ and $m^{2}>0$ and for these values of the masses the configuration $\phi_{1 c}=\phi_{3 c}=0$ minimizes the action. The AF and SC states have $m_{p}^{2}<0$ $\left(\phi_{3 c} \neq 0\right)$ and $m^{2}<0\left(\phi_{1 c} \neq 0\right)$, respectively. The $T=0$ transitions between the different phases, which are tuned varying the masses, are up to the classical level all continuous, second order transitions. The different phase diagrams, at mean field leve ${ }^{6.17 .30}$ were shown already in Fig. 1] However, renormalization group studies of the classical action yield that a bicritical point, as that of Fig. $1 \mathrm{~B}$ but occurring at finite temperatures, is stable only if the number of components of the superconductor $\left(n_{S}\right)$ and antiferromagnet $\left(n_{A F}\right)$ order parameter ${ }^{32}$ are such that $n=n_{S}+n_{A F} \leq 4$. For $n>4$ the $\mathrm{SO}(n)$ bicritical point at $T \neq 0$ becomes unstable and a phase diagram like that of Fig. 1C may occur. The above constraint implies, for example, that in a heavy fermion antiferromagnet of the Ising type, long range magnetic order can not coexist with superconductivity $\left(\phi_{1} \neq 0\right.$ and $\left.\phi_{3} \neq 0\right)$. No- 
tice that quantum corrections may destroy the classical $\mathrm{SO}(n)$ symmetry associated with the quantum bicritical point in Fig. 1B due to the different dynamics of the magnetic and superconductor fluctuations.

In the next sections, we study the quantum effects in two of the possible phase diagrams of Fig. 11 namely, cases (A) and (B). We begin considering case (A) where there is a normal phase separating the SC and AF phases and the transitions occur at different QCPs. The quantum effects become important in the normal region near both SC and AF phases where symmetry breaking and fluctuation induced quantum first order transitions can occur. We distinguish here between the case the system becomes antiferromagnetic in the presence of superconducting fluctuations from that where it becomes superconductor in the presence of antiferromagnetic fluctuations.

Case (C) of Fig. 11 with some additional assumptions, can also be described in our approach. In this case $\phi_{3}$ should be identified as the longitudinal component of the sub-lattice magnetization with a relaxational dynamics described by the propagator, Eq. (4). Also we have to neglect spin wave excitations which, at $T=0$, could be imagined as quenched if, for example, there is a sufficiently large anisotropy gap in their spectrum. In any case this situation has revealed up to now analytically intractable. Although it is possible to perform an expansion of the last logarithmic term in Eq. (14) in powers of $u$, this would be incompatible with the one-loop expansion which sums all powers of this quantity when performing the relevant integrals analytically, as we do below.

\section{QUANTUM EFFECTS IN THE NORMAL PHASE BETWEEN THE AF AND SC-QCPS}

\section{A. Fluctuation effects in the normal phase near superconductivity}

We are interested here in the superconducting-normal quantum phase transition and therefore, near the SC state, we look for a partially symmetry broken phase with $\phi_{1 c} \neq 0$ but $\phi_{3 c}=0$. In this case $D\left(\phi_{1 c}, \phi_{3 c}\right)=0$ and the quantum correction given by Eq. (14) can be written as

$$
\begin{aligned}
V^{(1)}\left(\phi_{1 c}\right)= & \frac{1}{2} \int \frac{d^{4} k}{(2 \pi)^{4}} \ln \left(1+\frac{(\lambda / 6) \phi_{1 c}^{2}}{k^{2}+m^{2}}\right)+ \\
& +\frac{1}{2} \int \frac{d^{4} k}{(2 \pi)^{4}} \ln \left(1+\frac{(\lambda / 2) \phi_{1 c}^{2}}{k^{2}+m^{2}}\right)+ \\
+ & \frac{1}{2} \int \frac{d^{4} k}{(2 \pi)^{4}} \ln \left(1+\frac{2 u \phi_{1 c}^{2}}{|\omega| \tau+q^{2}+m_{p}^{2}}\right)
\end{aligned}
$$

The first two integrations depend only on the modulus of the four-dimensional vector $k$ and time enters as an extra dimension as we are dealing with a Lorentz invariant case. This arises since the QCP of the superconductor transition (SQCP) has an associated dynamic exponent $z=1$. Therefore a cut-off regularization can be done as usual ${ }^{12}$. However, in the last integration we have anisotropy between time and space since the dynamic exponent which characterizes the scaling of time takes the value $z=2$. Hence, if we use a cut-off $\Lambda$ to the momentum, the correspondent frequency cut-off ${ }^{13}$ must be $\Lambda^{z}=\Lambda^{2}$. The integrations in Eq. (20) can be easily performed and the results expanded in powers of the parameter $m^{2}$ supposing proximity to the superconductor transition $\left(m^{2} \approx 0\right)$. The renormalization is done beginning from the massless case $m^{2}=0$ and generalized for small $\mathrm{m}^{2}$ following closely the renormalization procedure for the charged superfluid 22 . The effective potential

$$
V_{e f}\left(\phi_{1 c}, \phi_{3 c}=0\right)=V_{c l}\left(\phi_{1 c}, \phi_{3 c}=0\right)+V^{(1)}\left(\phi_{1 c}\right)
$$

where $V_{c l}$ is the classical potential given by Eq. [6], is

$$
V_{e f}\left(\phi_{c}\right) \approx \frac{1}{2} m^{2} \phi_{1 c}^{2}+\frac{\lambda}{4 !} \phi_{1 c}^{4}+\frac{\pi^{2}}{(2 \pi)^{4}}\left[\frac{8}{15}(2 u)^{5 / 2} \phi_{1 c}^{5}-\frac{8}{3}(2 u)^{5 / 2}\langle\phi\rangle \phi_{1 c}^{4}+\frac{4}{3}(2 u)^{3 / 2} m_{p}^{2} \phi_{1 c}^{3}-\frac{8}{3} m_{p}^{3} u \phi_{1 c}^{2}\right]
$$

Terms proportional to $\lambda^{2}$ and $m^{2} \lambda$ were neglected since they must be much smaller than the classical term proportional to $\lambda$ in the small coupling limit. The effective potential, Eq. (22), contains only the lowest order term in $m_{p}^{2}$ since we are near both transitions and consequently $m_{p}$ is also small. The term between brackets is the first quantum correction of order $\hbar$. As usual, the effective potential can be written as a function of its extremum, $\langle\phi\rangle$, which determines if the system is in the normal or in the broken symmetry superconducting phase. Higher powers in $u$ are not neglected since we have no classical term proportional to $u$. Consequently, even if $m^{2}>0$, balancing the classical $\lambda$ term with the $u$ terms of the quantum correction, it is possible to obtain asymmetric 
minima for $\phi_{1 c}$, i.e., a symmetry breaking in the normal state induced by the coupling $u$. For small masses $m_{p}$, independently of the value of $u$, the asymmetric minima are close to the origin $\phi_{1 c}=0$, as we can show by numerical inspection of the full effective potential (this also justifies the expansion for small $m_{p}^{2}$ ). Furthermore, with all minima close to the origin, we can neglect $\phi^{5}$ terms that cause instabilities to the potential (fortunately these terms become relevant far from the origin and hence away from the region of validity of the loop expansion).

For convenience, in Eq. (22) we have introduced the

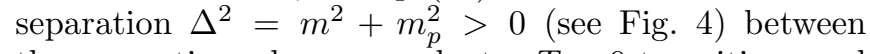
the magnetic and superconductor $T=0$ transitions and study the phase diagram as $\Delta^{2}$ is reduced. Notice that $\Delta^{2}$ measures the distance between the second order mean field QCPs in Fig. 1 and is also taken as a small quantity besides $m^{2}$ and $m_{p}^{2}$. Now, studying the minima of the effective potential we see that the quantum corrections induce symmetry breaking and the region where superconductivity is found in the phase diagram is extended. The shift of the superconductor QCP occurs towards the antiferromagnetic quantum critical point (AF$\mathrm{QCP}$ ), but in the paramagnetic phase. The analysis of the extrema of the potential can be easily carried out and has been presented before $\frac{15}{\underline{15}}$. We also find that the quantum fluctuations changes the nature of the superconducting transition to a first order one. The latent heat and spinodal points for this transition have also been calculated $^{15}$. Between the two spinodals there is an interval of coexistence of the superconducting phase with regions of strong antiferromagnetic fluctuations due to the occurrence of metastable minima.

\section{B. Fluctuation effects in the normal phase near the AF-QCP}

We follow the same procedure here as in the last section. Now we are interested in the AF quantum phase transition and therefore we look for a partially symmetry broken phase with $\phi_{3 c} \neq 0$ but $\phi_{1 c}=0$. We have once again $D\left(\phi_{1 c}, \phi_{3 c}\right)=0$ in the quantum correction given by Eq. (14) and the three resulting integrals have the same functional form of Eq. (20) but with different $\phi_{c}$ dependence. Integration and renormalization can be done as before beginning from the $m^{2}=0$ case. The full effective potential

$$
V_{e f}\left(\phi_{1 c}=0, \phi_{3 c}\right)=V_{c l}\left(\phi_{1 c}=0, \phi_{3 c}\right)+V^{(1)}\left(\phi_{3 c}\right)
$$

is similar to that obtained in the previous section except for the renormalization counterterms. Terms proportional to $u^{2}$ or $\lambda^{2}$ in the quantum corrections can not be neglected since there are no $\lambda$ or $u$ terms in the classical potential $\left(\phi_{1 c}=0\right)$. On the other hand, there is a classical coupling $g$ and higher order terms in $g$ can be discarded. Notice that for $m^{2}=0$ the result is the same as for the charged superfluid ${ }^{12,22}$,

$$
\begin{aligned}
V_{e f}\left(\psi_{c}, m\right. & =0) \approx \frac{1}{2}\left(m_{p}^{2}-\frac{m_{p}^{3} g}{12 \pi^{2}}\right) \phi_{3 c}^{2}+\frac{g}{4 !} \phi_{3 c}^{4}+ \\
& +\frac{u^{2}}{8 \pi^{2}} \phi_{3 c}^{4}\left[\ln \left(\frac{\phi_{3 c}^{2}}{\left\langle\phi_{3}\right\rangle^{2}}\right)-\frac{25}{6}\right]
\end{aligned}
$$

and therefore there is a fluctuation induced quantum first order transition varying $m_{p}^{2}$ for $g \sim u^{2}$. However, the correct results in the present case must be obtained for $m^{2} \neq 0$ where superconducting fluctuations are important but not critical. The effective potential to lowest order in powers of $m^{2}$, which is also small but finite, is given by,

$$
\begin{aligned}
V_{e f} & \approx \frac{1}{2} M_{p}^{2} \phi_{3 c}^{2}+\frac{g}{4 !} \phi_{3 c}^{4}+\frac{u^{2}}{8 \pi^{2}} \phi_{3 c}^{4}\left[\ln \left(\frac{\phi_{3 c}^{2}}{\left\langle\phi_{3}\right\rangle^{2}}\right)-\frac{25}{6}\right]+ \\
& +\frac{u m^{2}}{16 \pi^{2}}\left[\phi_{3 c}^{2}+2 \phi_{3 c}^{2} \ln \left(\frac{2 u \phi_{3 c}^{2}}{\Lambda^{2}}\right)\right]+\mathcal{O}\left(m^{4}\right)
\end{aligned}
$$

where $M_{p}^{2}$ is a renormalized magnetic mass parameter

$$
M_{p}^{2}=m_{p}^{2}-\frac{m_{p}^{3} g}{12 \pi^{2}} .
$$

We have now in Eq. (25) a new term proportional to $u m^{2}$ and since it depends on the cut-off $\Lambda$ another counterterm is needed. With a minimum counterterm we get,

$$
\begin{aligned}
V_{e f}\left(\phi_{3 c}\right) & \approx \frac{1}{2}\left(M_{p}^{2}+\frac{9 u^{2}\left\langle\phi_{3}\right\rangle^{2}}{2 \pi^{2}}-\frac{1}{2} g\left\langle\phi_{3 c}\right\rangle^{2}\right) \phi_{3 c}^{2}+ \\
& +\frac{g}{4 !} \phi_{3 c}^{4}+\frac{u^{2}}{8 \pi^{2}} \phi_{3 c}^{4}\left[\ln \left(\frac{\phi_{3 c}^{2}}{\left\langle\phi_{3}\right\rangle^{2}}\right)-\frac{25}{6}\right]+ \\
& +\frac{u m^{2}}{8 \pi^{2}} \phi_{3 c}^{2}\left[\ln \left(\frac{\phi_{3 c}^{2}}{\left\langle\phi_{3}\right\rangle^{2}}\right)-3\right]
\end{aligned}
$$

where other small quadratic terms in the field have been included in the renormalized mass $M_{p}^{2}$. The coupling to the massive superconductivity fluctuations changes dramatically the behavior of the potential since now its second derivative at $\phi_{3 c}=0$ is always negative, i.e., the origin is always a maximum for any $u m^{2} \neq 0$. This coupling also gives rise to new minima near $\phi_{3 c}=0$ which move away from the origin as its strength is increased (Fig. 3). It is interesting that as $M_{p}^{2}$ is reduced, i.e., as the system moves away from the SC-QCP towards the AF-QCP, it goes, through a quantum first order transition at $M_{p}^{c 2}$, from a phase with a reduced value of the antiferromagnetic order parameter to another with a larger value of $\phi_{3 c}$ (Figs. [3] and [4). The AF phase closest to the superconductor is always the small moment one.

In Fig. 5 the ratio $R$ of the sub-lattice magnetizations in the small moment antiferromagnetic (SMAF) and large moment antiferromagnetic (LMAF) phases is plotted as a function of the relevant parameters, namely the ratio $u^{2} / g$. Small moment antiferromagnetism (SMAF) is a common feature in heavy fermion materials ${ }^{33}$. The 
most common values for the ratio $R$ observed experimentally 33.34 are of order $10^{-2}, 10^{-3}$ and correspond to values of $u^{2} / g \approx 1$. We have found that the ratio $R$ is independent of the values of $g$ and $\Delta$ being a function exclusively of the scaled variable $u^{2} / g$.

There is an interesting similarity between the phase diagram of the heavy fermion $Y b R h_{2} S i_{2}$, for which a first order phase transition from a low moment AF phase to a large moment magnetic phase has been observed with increasing pressure ${ }^{34}$ and the results obtained above. In $Y b$ based heavy fermions, pressure $(\mathrm{P})$ acts on the opposite direction it does on $\mathrm{Ce}$ compounds decreasing the ratio $J / W$ between the Kondo lattice parameters ${ }^{10}$. $Y b R h_{2} S i_{2}$ at $P=0$ is a SMAF system with $T_{N}=70 \mathrm{mk}$. Pressure increases $T_{N}$ and at $P_{c} \approx 10 G P a$ there is a first order transition to a high moment state with $\mu_{Y b} \approx 1.9 \mu_{B}$. On the other hand, negative pressure, i. e., expansion of the lattice drives this system to an antiferromagnetic quantum critical point $\mathrm{H}^{35}$. It is an exciting possibility that further expansion of the lattice would give rise to superconductivity.

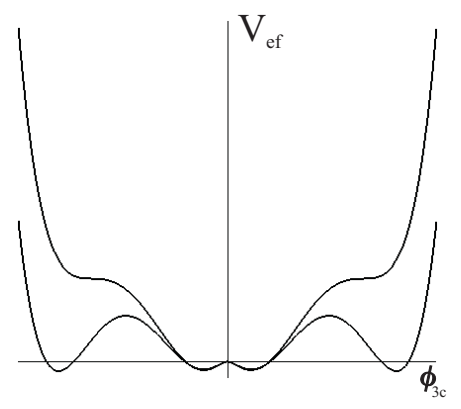

FIG. 3: New minima appear in the potential for $u m^{2} \neq 0$. The effective potential is shown here for two situations: for $M_{p}^{2}=M_{p}^{c 2}$, where the first order transition from LMAF to SMAF occurs and these two states become degenerate and for the spinodal point at which the LMAF becomes unstable inside the SMAF phase.

When the separation $\Delta^{2}$ between the classical $T=0$ critical points is reduced the magnetic moment in the SMAF goes continuously to zero. In practice, when the separation is small enough, the moment in the SMAF is so low that it can be identified with the normal state. Then for $\Delta^{2}$ sufficiently small, but still large enough to avoid the possibility of a direct transition from $\mathrm{AF}$ to $\mathrm{SC}$, there is a first order AF-normal transition. The spinodal points and the energy equivalent to the latent heat can be calculated exactly as in the charged superfluid ${ }^{22.36}$ since the term proportional to $u m^{2}$ can be neglected. Particularly, the effective potential at the asymmetric minimum close to the first order transition can be written as

$$
V_{e f f}\left(\left\langle\phi_{3 c}\right\rangle\right) \approx \frac{1}{4} M_{p}^{2}\left\langle\phi_{3}\right\rangle^{2}\left[1-\frac{m_{c}^{2}}{M_{p}^{2}}\right]
$$
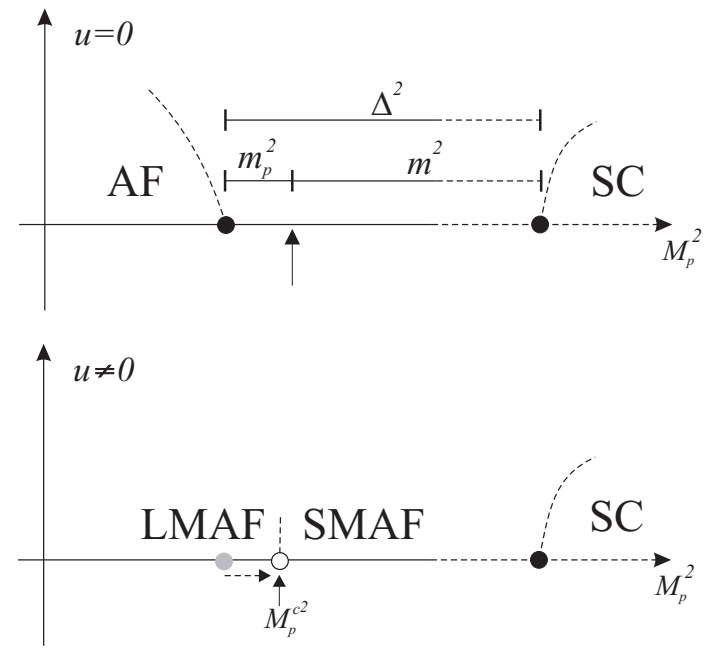

FIG. 4: Phase diagrams for $u=0$ and $u \neq 0 . \Delta^{2}$ is the distance between the AF and SC QCPs. $m_{p}^{2}$ and $m^{2}$ are the distances from the point the system is actually probed to the $\mathrm{AF}$ and SC QCPs respectively. When $M_{p}^{2} \approx m_{p}^{2}$ is reduced, i.e., the system moves away from the SC-QCP, there is a first order transition at $M_{p}^{c 2}$ between two AF phases with different values of the order parameter.

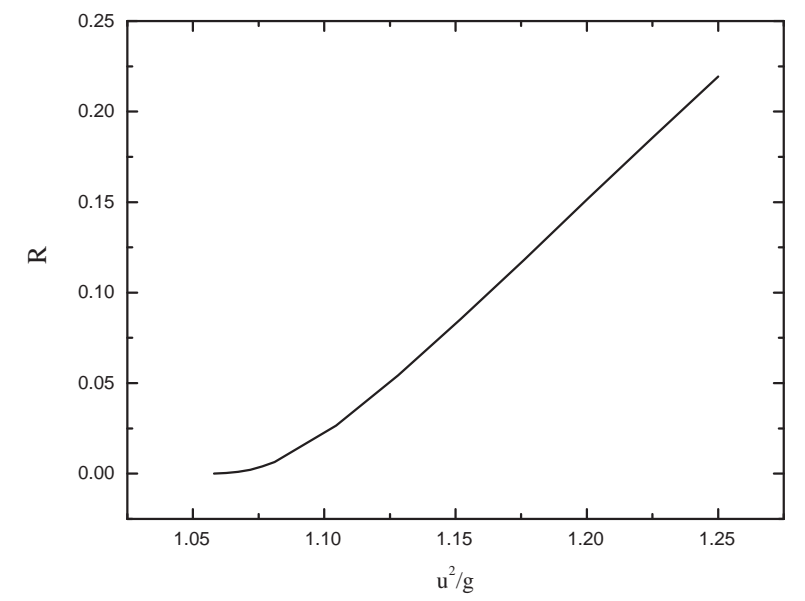

FIG. 5: The ratio $R=\left\langle\phi_{c}^{S M}\right\rangle /\left\langle\phi_{c}^{L M}\right\rangle$ between the ground state sub-lattice magnetizations in the phases SMAF and LMAF as a function of $g^{2} / u$.

where the critical mass $m_{c}^{2}$ is

$$
m_{c}^{2}=\frac{3 u^{2}}{12 \pi^{2}}\left\langle\phi_{3}\right\rangle^{2}
$$

Therefore, the latent heat is given by the simple expres- 
sion

$$
L_{h}=\frac{1}{4} m_{c}^{2}\left\langle\phi_{3}\right\rangle^{2}
$$

A similar phenomenon occurs for the first order SMAFLMAF transition. In this case there is also an associated latent heat and the spinodal point corresponding to the limit of stability of the large moment phase into the SMAF phase is shown in Fig. 3

\section{QUANTUM BICRITICAL POINT}

In this section we study the possibility of a quantum bicritical point (QBP) as shown in Fig 1B. The quantum corrections can be calculated in the paramagnetic side and involve terms of higher order in the couplings $u, \lambda$ and $g$. In the limit of small coupling considered in this work, these corrections are then of little importance, especially for the quantum transition, since the classical part becomes much larger. However, mass renormalization can change the properties of the superconductor itself as, for example, it affects the constant $\kappa$ which determines whether the system is a type I or type $\mathrm{II}^{15}$.

The transition where both phases collapse is continuous, as obtained classically and the analysis of this double critical point can be done more easily using a quantum scaling theory ${ }^{37}$. Classically this point has $\mathrm{SO}(n)$ symmetry ${ }^{17}$, but when taking into account its quantum character, as it occurs at $T=0$, there are different dynamics related to the distinct phases on both sides of the transition. It is easy to convince oneself that in the low frequency, long wavelength limit, it is the slow relaxation dynamics of the magnetic component with the associated $z=2$ dynamic exponent that is relevant for the critical behavior. For $d=3$, assuming that a unique correlation length diverges as $T$ is reduced at the QBP, we obtain that both the antiferromagnetic $\left(T_{N}\right)$ and superconducting $\left(T_{S}\right)$ critical lines of finite temperature phase transitions rise with the distance $\delta$ to the QBP, as $T_{N, S} \propto|\delta|^{\psi}$, i.e., with the same shift exponent $\psi$. Furthermore, we find $\psi=\nu z$, where $\nu$ and $z$ are the correlation length and the dynamic exponents associated with the QBP. Since the effective dimension $d_{\text {eff }}=d+z=5$, we have $\nu=1 / 2$, such that the quantum bicritical crossover exponent ${ }^{37} \nu z=1$. Alternatively, the critical line can be written as $\delta(T)=\delta(T=0)+u T^{1 / \psi}$. For the correlation length along the critical trajectory, i.e., for $\delta(T=0)=0$ scaling yields

$$
\xi \sim|\delta(T)|^{-\nu} \stackrel{\delta(0)=0}{\longrightarrow} \xi \sim T^{-\nu / \psi}=T^{-1 / 2}
$$

so that at the QBP the correlation length diverges with decreasing temperature as $1 / \sqrt{T}$. The scaling of other physical quantities can be predicted similarly, particularly, the specific heat along this line has a non-Fermi liquid, $C_{P}(T) \propto \sqrt{T}$, behavior ${ }^{37}$ (see Fig. 6).

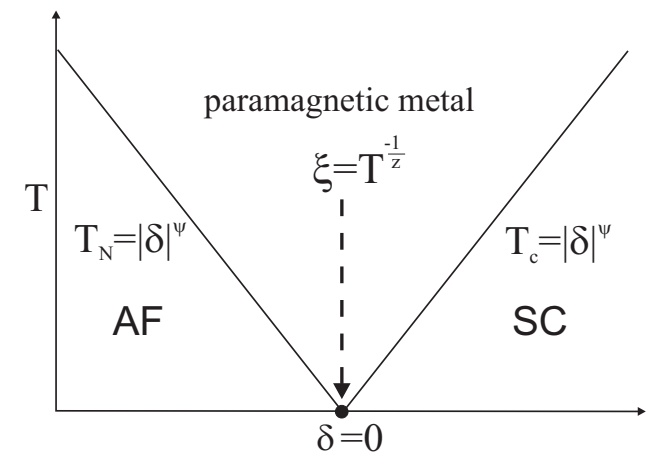

FIG. 6: A quantum bicritical point separating an antiferromagnet from a superconductor. Both lines of finite temperature phase transitions rise with the same exponent $\psi$. For $\mathrm{d}=3, \psi=\nu z=1$.

\section{CONCLUSIONS}

In this paper we have considered a generalized Ginzburg-Landau functional including both superconducting and magnetic order parameters to study the mutual influence of these instabilities in three dimensional heavy fermion metals at zero temperature. The quantum corrections to this classical functional were obtained using the effective potential method up to one loop order. This method had to be generalized to take into account the dissipative nature of the propagator of the paramagnons in the metallic paramagnetic phase close to the antiferromagnetic instability. The superconductor propagator we used corresponds to the simplest generalization of the Ginzburg-Landau classical action to take into account dynamic or quantum effects. The calculations however can be carried out with different propagators, for example, that of Ramazashvili and Coleman ${ }^{23}$. The results will be presented elsewhere. We point out that the exact form of the superconductor propagator is irrelevant for the qualitative results of sections VI-A and VII.

Different phase diagrams have been considered according to the distance between the quantum critical points in the classical potential. For non-coincident QCPs the second order nature of the quantum transitions obtained at the classical level is modified by the quantum corrections, changing from continuous to weak first order transitions. Hence, the spin fluctuations can change the nature of the superconducting transition in the same way that the coupling to a magnetic field does ${ }^{22,38}$. For this fluctuation induced first order transition we can calculate the equivalent of the latent heat to transitions at $T=0$ and the spinodal points. Both indicate that we are dealing with a weak first order transition as they are directly related to the small coupling constant $u$.

We also have found that a metallic antiferromagnetic QCP is strongly affected by the presence of superconducting fluctuations. These lead to the appearance of a 
small moment phase which goes through a weak first order transition to a large moment one as the system moves away from the superconductor instability.

Finally, in the case of coincident quantum critical points the transition remains second order and the finite temperature behavior can be extracted from the scaling properties of the quantum bicritical point.

The effects of the competition between the AF and SC instabilities are highly non-trivial since a positive coupling which classically tends to avoid the coexistence of these states leads when quantum corrections are considered to symmetry breaking in the normal state and increases the region of the ordered phases (SC and $\mathrm{AF}$ ) in the phase diagram.

In recent years increasing experimental evidence is being gathered that points to existence of intrinsic inhomogeneities close to the $T=0$ antiferromagnetic instabil- ity in heavy fermions ${ }^{16}$. Coexistence of small ordered moments with paramagnetic regions, coexistence between superconductivity and paramagnetism have been observed. The present approach provides an appealing model for this type of phenomena. They arise in our theory as a direct consequence of the competition between different order parameters which give rise, through quantum corrections, to weak first order transitions with the associated physics of metastability, spinodal points and phase coexistence.

\section{Acknowledgments}

The authors would like to thank the Brazilian Agencies, FAPERJ and CNPq for financial support.
* Electronic address: andre@if.uff.br,mucio@if.uff.br

1 R. Nagarajan, C. Mazumdar, Z. Hossain, S. K. Dhar, K. V. Gopalakrishnan, L. C. Gupta, C. Godart, B. D. Padalia and R. Vijayaraghavan, Phys. Rev. Lett. 72, 274 (1994).

2 B. K. Cho, P. C. Canfield, and D. C. Johnston, Phys. Rev. Lett. 77, 163 (1996).

${ }^{3}$ H. Hegger, C. Petrovic, E. G. Moshopoulou, M. F. Hundley, J. L. Sarrao, Z. Fisk and J. D. H. Thompson, Phys. Rev. Lett. 84, 4986 (2001).

4 J. D. Thompson, R. Movshovich, Z. Fisk, F. Bouquet, N. J. Curro, R. A. Fisher, P. C. Hammel, H. Hegger, M. F. Hundley, M. Jaime, P. G. Pagliuso, C. Petrovic, N. E. Phillips and J. L. Sarrao, J. Magn. Mater 226, 5 (2001).

${ }^{5}$ C. Petrovic, R. Movshovich, M. Jaime, P. G. Pagliuso, M. F. Hundley, J. L. Sarrao, Z. Fisk and J. D. Thompson, Europhys. Lett. 53, 354 (2001).

${ }^{6}$ N. D. Mathur, F. M. Grosche, S. R. Julian, I. R. Walker, D. M. Freye, R. K. W. Haselwimmer and G. G. Lonzarich, Nature 394, 39 (1998).

7 H.J. Kang, P. C. Dai, J. W. Lynn, M. Matsuura, J. R. Thompson, S. C. Zhang, D. N. Argyriou, Y. Onose and Y. Tokura, Nature, 423, 522 (2003)

${ }^{8}$ F. Steglich, J. Aarts, C. D. Bredl, W. Lieke, D. Meschede, W. Franz and H. Schafer, Phys. Rev. Lett., 43, 1892 (1979); S. Raymond and D. Jaccard, Phys. Rev. B 61, 8679 (2000).

9 M. A. Continentino, G. Japiassu and A. Troper, Phys. Rev. B39 , R9734 (1989).

10 M. A. Continentino, Phys. Rev. B 47, 11587 (1993).

11 G. Jona-Lasinio, Nuovo Cimento 34, 1790 (1964).

12 S. Coleman and E. Weinberg, Phys. Rev. D 7, 1888 (1973).

13 J. A. Hertz, Phys. Rev. B 14, 1165 (1976).

14 A. J. Millis, Phys. Rev. B48, 7183 (1993).

15 A. S. Ferreira, M. A. Continentino and E. C. Marino, Solid St. Comm. 130, 321 (2004).

16 J. Flouquet, Y. Haga, P. Haen, D. Braithwaite, G. Knebel, S. Raymond and S. Kambe, cond-mat/0306157 and private communication; N. Bernhoeft, J. Phys.:Cond.Mat, 13, R771 (2001).

17 S. C. Zhang, Science 275, 1089 (1997).

18 S. Sachdev and S.C. Zhang, Science 295, 452 (2002); E.
Demler, S. Sachdev and Y. Zhang, Phys. Rev. Lett. 87, 067202 (2001).

19 H. Kohno, H. Fukuyama and M. Sigrist, J. Phys. Soc. Jpn. 681500 (1999).

20 G. Baskaran and P. W. Anderson, J. Phys. Chem. solids 59, $1780(1998)$

21 X. Hu, Phys. Rev. Lett. 87, 057004 (2001); A. Aharony, Phys. Rev. Lett. 88, 059703 (2002); X. Hu, Phys. Rev. Lett. 88, 059704 (2002); M. Jostingmeier, A. Dorneich, E. Arrigoni, W. Hanke, S. C. Zhang, cond-mat/0207528 (2002)

22 See M. A. Continentino, Quantum Scaling in Many-Body Systems, World Scientific, Singapore, (2001), chapter 12.

23 R. Ramazashvili and P. Coleman, Phys. Rev. Lett. 79, 3752 (1997)

${ }^{24}$ T. R. Kirkpatrick and D. Belitz, Phys. Rev. Lett. 79, 3042 (1997).

25 T. Timusk and B. Statt, Rep. Prog. Phys. 62, 61-122 (1999)

26 T. Ekino, T. Takabatake, H. Tanaka and H. Fujii, Phys. Rev. Lett. 75, 4262 (1995); S. Donovan, A. Schwartz and G. Grner Phys. Rev. Lett. 79, 1401-1404 (1997); D. N. Davydov, S. Kambe, A. G. M. Jansen, P. Wyder, N. Wilson, G. Lapertot and J. Flouquet, Phys. Rev. B 55, R7299R7302 (1997); M. Dressel, N. Kasper, K. Petukhov, B. Gorshunov, G. Grner, M. Huth, and H. Adrian, Phys. Rev. Lett. 88, 186404 (2002); V. A. Sidorov, M. Nicklas, P. G. Pagliuso, J. L. Sarrao, Y. Bang, A. V. Balatsky and J. D. Thompson, Phys. Rev. Lett. 89, 157004 (2002); V. A. Sidorov, M. Nicklas, P. G. Pagliuso, J. L. Sarrao, Y. Bang, A. V. Balatsky and J. D. Thompson, Phys. Rev. Lett. 89 157004 (2002).

27 R. Micnas, J. Ranninger and S. Robaszkiewicz, Rev. Mod. Phys. 62, 113 (1990)

28 J. A. Hertz and M. A. Klenin, Phys. Rev. B 10, 1084 (1974)

29 J. Lindhard, Dan. Vidensk. Selsk. Mat.-Fys. Medd. 288 (1954);

30 P. G. Pagliuso, N. O. Moreno, N. J. Curro, J. D. Thompson, M. F. Hundley, J. L. Sarrao, Z. Fisk, A. D. Christianson, A. H. Lacerda, B. E. Light and A. L. Cornelius., Phys. Rev. B66, 054433 (2002); M. Nicklas, V. A. Sidorov, H. A. 
Borges, P. G. Pagliuso, C. Petrovic, Z. Fisk, J. L. Sarrao, and J. D. Thompson, Phys.Rev. B67, 020506 (2003); R. A. Fisher, F. Bouquet, N. E. Phillips, M. F. Hundley, P. G. Pagliuso, J. L. Sarrao, Z. Fisk, and J. D. Thompson, Phys.Rev. B65, 224509 (2002).

31 S. Coleman, Aspects of Symmetry, Cambridge University Press (1985) chapter 5.

32 J. M. Kosterlitz, D. R. Nelson and M. F. Fisher, Phys. Rev. B13, 412 (1976).

33 W. J. L. Buyers, Physica B223\&224, 9 (1996).

34 J. Plessel, M. M. Abd-Elmeguid, J. P. Sanchez, G. Knebel, C. Geibel, O. Trovarelli and F. Steglich, Phys. Rev. B67,
180403(R) (2003).

35 J. Custers, P. Gegenwart, H. Wilhelm, K. Neumaier, Y. Tokiwa, O. Trovarelli, C. Geibel, F. Steglich, C. Pepin and P. Coleman, Nature 424, 524 (2003).

36 M. A. Continentino and A. S. Ferreira, Physica A 339, 461 (2004).

37 See M. A. Continentino, Quantum Scaling in Many-Body Systems, World Scientific, Singapore, (2001), chapter 1.

38 B. I. Halperin, T. C. Lubensky and Shang-keng Ma, Phys. Rev. Lett. 32, 292 (1974). 